\title{
二次有机气溶胶估算方法比较研究
}

\author{
郭松胡敏* 郭庆丰尚冬杰 \\ (北京大学环境模拟与污染控制国家重点实验室 北京大学环境科学与工程学院 北京 100871)
}

\begin{abstract}
摘要 为研究北京夏季二次有机气溶胶 (SOA)浓度, 比较多种 SOA 估算方法的不确定性和在我国的适用性, 在 CAREBEIJING 2008 大型观测期间在城市点北大点和郊区点榆牮点采集大气颗粒物 $\mathrm{PM}_{2.5}$ 样品, 利用多种方法对二次 有机碳(SOC)的浓度进行了估算, 估算方法包括二次有机示踪物产率法、非一次源 OC 法、非生物质燃烧水溶性有机碳 法(WSOC)和元素碳示踪有机碳/元素碳比值法. 通过模拟我国典型的生物质燃烧, 建立适合我国的非生物质燃烧 WSOC 方法, 获得方法中重要参数生物质燃烧排放颗粒物中 WSOC/OC 的比值, 我国生物质燃烧颗粒物中该比值平均 为 $0.48 \pm 0.04$. 多种方法的结果均表明, SOA 已经成为北京颗粒有机物的重要组成部分, 所占比例可以达到 $50 \%$ 甚至更 高. 利用化学质量守恒模型 $(\mathrm{CMB})$ 和示踪物产率法对颗粒有机物来源进行闭合发现，依靠目前的示踪物技术北京大气 颗粒有机物中仍然有 $20 \% \sim 27 \%$ 的来源不清楚. 通过综合比较了多种 SOC 估算方法, 确定了各方法在我国的适用性: 示踪物产率法、非一次源 OC 法和 EC 示踪 OC/EC 比值法可适用于我国大气中 SOC 的估算, 但是非生物质燃烧 WSOC 方法仅适用于我国大气中水溶性 SOC 的估算. 本研究还对这几种方法的不确定性做了初步的分析, 为其他研究者选择 估算方法提供参考和依据. 示踪物产率法仅估算了几种特定 VOCs 前体物对 SOC 贡献, 因此低估了总的 SOC; 同样地, 非生物质燃烧 WSOC 法仅估算了水溶性的 SOC, 因此也低估了总的 SOC; 非一次源 OC 法高估了 SOC, 这主要是由于 未能解析出的一次源 $\mathrm{OC}$ 造成的. 而且这种高估在城市地区更为显著, 主要是因为城市地区一次源相对比较复杂造成 的; EC 示踪 OC/EC 比值法的主要不确定性来自于一次源 OC/EC 比值的确定, 由于这个比值造成的单点误差最高可高 估 $54 \%$ 或低估 $64 \%$.
\end{abstract}

关键词 二次有机气溶胶; 二次有机碳; 估算方法; 方法适用性; 比较研究

\section{Comparison of Secondary Organic Aerosol Estimation Methods}

Guo, Song Hu, Min* Guo, Qingfeng Shang, Dongjie

(State Key Joint Laboratory of Environmental Simulation and Pollution Control, College of Environmental Sciences and Engineering, Peking University, Beijing 100871)

Abstract To investigate the secondary organic aerosol (SOA) formation in Beijing, fine particle $\left(\mathrm{PM}_{2.5}\right)$ samples were collected at an urban site (Peking University, PKU) and a rural site (Yufa) during CAREBEIJING 2008 summer intensive field campaign. Several approaches were used to estimate SOA concentrations, including tracer-yield method, non-primary organic carbon method (receptor model, Chemical Mass Balance model), non-biomass burning water soluble organic carbon (WSOC) method and EC tracer OC/EC ratio method. To develop non-biomass burning WSOC method, simulation of typical Chinese biomass burning was conducted to obtain the important parameters. The average WSOC/OC ratio in Chinese biomass burning is $0.48 \pm 0.04$. This ratio can be used in non-biomass burning WSOC method to estimate SOC of China. The results from different methods all indicated secondary formation has become major contributor to organic aerosols in Beijing, accounting for $50 \%$ or more of the total OC. A closure study was made by combination of CMB model and tracer-yield method. Five primary sources, including vegetative detritus, biomass burning, coal burning, gasoline engines and diesel engines, and four secondary organic aerosols derived from isoprene, $\alpha$-pinene, $\beta$-caryophyllene and toluene were apportioned. To the current knowledge, about $20 \% \sim 27 \%$ of the OC sources still remain unknown. Applicability of these SOC methods in China was tested by comparing the different methods. Tracer-yield method, CMB model and EC tracer OC/EC ratio method can be used to estimate SOC in China. However, non-biomass burning WSOC method can only be used to estimate water-soluble SOC. Uncertainty analysis was conducted to help researchers to determine the proper method to estimate SOC in China. Tracer-yield method underestimates total SOC, because it can only estimate SOCs from several precursors. Similarly, non-biomass burning WSOC method also underestimate SOC. Non-primary OC method overestimates SOC due to unapportioned primary OC, especially in urban area where the particle sources are complicated. The uncertainty of EC-tracer OC/EC ratio method is mainly from the primary $\mathrm{OC} / \mathrm{EC}$ ratio. The largest overestimation and underestimation of single point value can be $54 \%$ and $64 \%$.

* E-mail: minhu@pku.edu.cn

Received April 14, 2014; published May 11, 2014.

Project supported by the National Basic Research Program of China (No. 2013CB228503) and the National Natural Science Foundation of China (Nos. 21025728, 21190052, GZ663, 41121004).

项目受国家重点基础研究发展计划(No. 2013CB228503)和国家自然科学基金(Nos. 21025728, 21190052, GZ663, 41121004)资助. 
Keywords secondary organic aerosol; secondary organic carbon; estimation method; method applicability; comparison study

\section{1 引言}

二次有机气溶胶(SOA) 是指大气中的挥发性有机物 (VOCs) 经过光化学氧化生成的颗粒有机物 ${ }^{[1]}$. SOA 是颗 粒有机物的重要组成部分, 全球模型的结果表明, 在中 纬度地区, SOA 平均可以占到总颗粒有机物的一半左

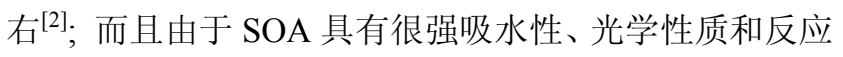
活性等特点, 因此对区域空气质量和人体健康、全球气 候变化均有重要影响 ${ }^{[1,3]}$. 但是, 由于 SOA 前体物 VOCs 的种类和来源复杂多样, 而且这些 VOCs 在大气中经历 着非常复杂的化学反应 ${ }^{[4]}$, 因此人们对于 SOA 的认识还 非常有限, 甚至对其总量的估算都存在很大的误差. 另 外, $\mathrm{SOA}$ 生成也会参与到其他的大气化学过程, 例如新 粒子生成和气溶胶老化等 ${ }^{[5 \sim 8]}$, 人们对这些过程的机制 也十分不明确. 对于 SOA 认识的缺乏也被认为是目前 气候模型中不确定性的最主要来源 ${ }^{[9,10]}$. 弄清 SOA 的组 成、来源和生成机制已经成为认识大气复合污染和细颗 粒物环境效应的关键. 研究者们开发出多种方法对 SOA 进行估算, 包括离线膜采样的方法和在线的气溶 胶质谱(AMS)技术等 ${ }^{[11 ~ 14]}$. 国内的一些研究者也通过 模拟和理论计算等研究 SOA 的生成机制 ${ }^{[15 \sim 17]}$. Guo 等 ${ }^{[18]}$ 总结了 $\mathrm{SOA}$ 研究最主要的手段以及基于外场观测的 SOA 估算方法, 但是, 不同的方法之间存在着很大的差 异 ${ }^{[19]}$, 因此有必要对多种方法进行综合比较, 并对这些 方法在不同地区的适用性进行验证，从而为进一步的二 次有机气溶胶研究提供方法学的依据.

近年来, 随着经济的快速发展和城市化进程的不断 加剧, 我国面临着严重的大气污染问题, 尤其是在北 京、长三角和珠三角等超大城市和城市群地区. 而且我 国的大气污染日益表现出以强氧化性和高细颗粒物浓 度为特征的区域大气复合污染 ${ }^{[20,21]}$, 其中高浓度的 SOA 就是大气复合污染的重要表征 ${ }^{[11,14]}$. 因此有必要对我国 的 SOA 进行深入研究, 并且综合比较不同的估算方法, 探讨这些方法的不确定性以及在我国的适用性.

本研究在 CAREBEIJING 2008 大型观测期间采集 大气细颗粒物 $\mathrm{PM}_{2.5}$ 样品, 并利用多种方法对颗粒物中 的 SOC 进行估算, 包括二次有机示踪物产率法、非一次 源 OC 法、非生物质燃烧水溶性有机物法和元素碳示踪 $\mathrm{OC} / \mathrm{EC}$ 比值法, 并综合分析和比较多种方法的结果, 初 步探讨了各种方法的不确定性以及在我国的适用性, 为 研究者在选择估算方法对我国二次有机气溶胶进行研 究时提供参考和依据.

\section{2 结果与讨论}

\section{1 二次有机碳的估算方法}

对于 SOA 浓度的直接测量是比较困难的, 因此一
般对 SOA 中的碳含量也就是二次有机碳(SOC)进行估 算. 本研究利用四种方法对北京 2008 年夏季大气颗粒 物中的 SOC 进行了估算.

2.1.1 基于二次有机示踪物的示踪物产率法(Tracer Yield Method)

美国环境保护署(US EPA)最近开发了一种基于二 次有机示踪物的示踪物产率法用来估计二次源对大气 中颗粒有机物的贡献 ${ }^{[22 ~ 24]}$. Kleindienst 等 ${ }^{[23]}$ 利用烟雾箱 模拟几种生物源和人为源 VOCs 生成 SOA 的过程, 并对 生成的颗粒物进行分析, 生成的颗粒物总质量即为 $\mathrm{SOA}$ 的总质量 [SOA], 测定其中的总有机碳即为 [SOC]. 对收集到的气溶胶样品进行提取和化学衍生, 衍生后的 样品最后进行 GC-MS 分析, 分析 SOA 的主要成分, 以 此确定某一特定 VOCs 前体物生成 SOA 的示踪物, 并将 其定量. 目前已确定了异戊二烯、 $\alpha$-蒎烯、 $\beta$-丁香烯和 甲苯作为前体物生成 SOA 的示踪物. 通过测定示踪物 浓度和 SOA, SOC 总质量, 确定示踪物同 SOA、SOC 的 关系, 用 SOA 质量百分比 $f_{\mathrm{soa}, \mathrm{hc}}$ 和 SOC 质量百分比 $f_{\mathrm{soc}, \mathrm{hc}}$ 表示. 对于单一物种碳氢化合物 VOCs 前体物, 其对二 次有机气溶胶的质量百分比 $f_{\text {soa,hc }}$ 定义为:

$$
f_{\mathrm{soa}, \mathrm{hc}}=\frac{\sum_{i}\left[t_{i}\right]}{[\mathrm{SOA}]}
$$

其中, $\left[t r_{i}\right]$ 为第 $i$ 种示踪物的浓度, 单位 $\mu \mathrm{g} / \mathrm{m}^{3}, \mathrm{SOA}$ 质量 为烟雾箱中生成颗粒态物种的总质量, 利用这个公式可 以得到:

$$
f_{\text {soc,hc }}=f_{\text {soa,hc }} \frac{[\mathrm{SOA}]}{[\mathrm{SOC}]}
$$

这个公式可以用来计算 $\mathrm{SOC}$ 的质量百分比 $f_{\mathrm{soc}}$, 利 用这两个公式就可以得到 SOA 和 SOC 的质量百分比. 最后通过实际大气中测量各种前体物生成 SOA 的示踪 物的浓度, 结合 SOA 质量百分比 $f_{\mathrm{soa}, \mathrm{hc}}$ 和 SOC 质量百分 比 $f_{\mathrm{soc}, \mathrm{hc}}$, 即可得到不同前体物 VOCs 对 SOA 和 SOC 的 贡献.

本研究识别并定量了北京大气颗粒物中 14 种二次 有机示踪物，这些示踪物的前体物分别为生物源排放的 异戊二烯、 $\alpha$-蒎烯、 $\beta$-丁香烯(卡莱烯)和人为源排放的 甲苯. 表 1 给出了观测期间这些二次有机示踪物的平均 浓度. 利用示踪物浓度和示踪物产率法, 可以估算不同 VOCs 前体物对 SOA 的贡献. 图 1 给出了本研究得到的 异戊二烯、 $\alpha$-蒎烯、 $\beta$-丁香烯和甲苯生成 SOC 对总 $\mathrm{OC}$ 的贡献. 在北大点甲苯 SOC 是几种 SOC 中所占比例最 高的，可以占总 $\mathrm{OC}$ 的 $(16.6 \pm 8.4) \%$, 异戊二烯、 $\alpha$-蒎烯 和 $\beta$-丁香烯生成 SOC 分别占 $\mathrm{OC}$ 的 $(8.9 \pm 5.8) \%,(4.8 \pm$ $2.2) \%$ 和 $(2.2 \pm 1.9) \%$. 榆袋点甲苯 $\mathrm{SOC}$ 和异戊二烯 SOC 
是所占比例最高的，分别可以占到(14.1 \pm 8.5$) \%$ 和(13.9 $\pm 8.9) \%, \alpha$-蒎烯和 $\beta$-丁香烯生成 SOC 占 $\mathrm{OC}$ 的比例分别 为 $(5.7 \pm 1.9) \%$ 和 $(1.7 \pm 1.0) \%$. 值得关注的是甲苯 $\mathrm{SOC}$ 在榆牮点也占相当高的比例, 表明由于城市化进程的加 剧, 北京城市地区人为源污染对北京乡村地区也有很大 的影响. 综合以上结果, 示踪物产率法估算得到北大点

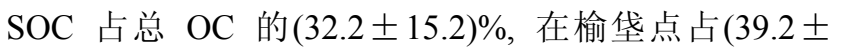
$14.8) \%$.

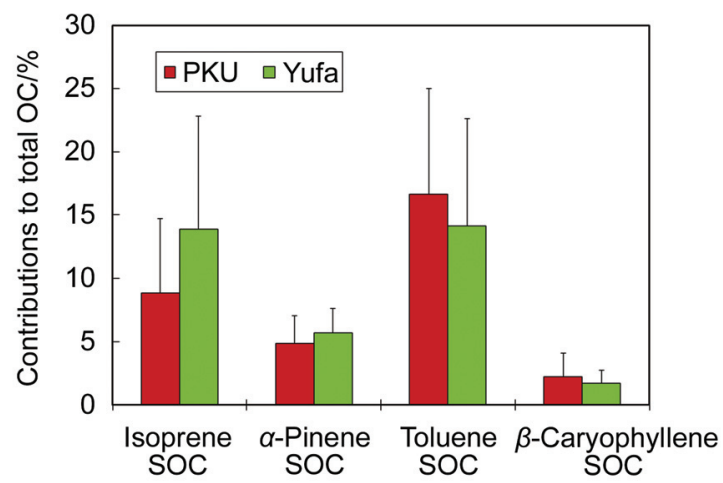

图 1 北大点和榆垈点生物源与人为源 SOC 对有机碳的贡献

Figure 1 Contributions of biogenic and anthropogenic SOC to total OC

\subsection{2 非一次源有机碳法(Non-primary OC)}

对于颗粒有机物一次源的准确解析是对颗粒有机 物二次源解析的基础. 随着颗粒物源解析技术的发展, 对颗粒有机物一次源解析的技术日趋完善. 在颗粒有机
物源解析技术中，受体模式已经成为一种较为成熟的技 术方法，而且在目前的源解析工作中得到了广泛的应用. 而其中 $\mathrm{CMB}$ 模型由于其物理意义明确, 因此被认为是 准确解析颗粒有机物一次源的重要工具之一 ${ }^{[25 ~ 27]}$. 由 于 $\mathrm{CMB}$ 模型的基本假设是从源到受体符合质量守恒, 因此无法直接对二次来源进行解析. 但是一般认为, 受 体模型未能解析出的部分主要来自二次生成, 利用总 $\mathrm{OC}$ 和解析出的一次源 OC 可以估计二次源对有机颗粒 物的贡献, 这种方法也称为非一次源 $\mathrm{OC}$ 法. 从原理上 可以看出，这种方法是非常粗略的估计，仅仅能对颗粒 有机物二次来源的贡献作为参考. 另外正交矩阵因子分 析模型(PMF)也是一种常用的用来解析颗粒有机物来源 的受体模型, 但是在本研究中并无涉及.

本研究利用 CMB 模型对颗粒有机物一次来源进行 解析, 选择了 5 个一次源, 包括植物碎屑、生物质燃烧、 柴油车、汽油车和燃煤, 示踪物包括 8 种烷烃、3 种蕉 烷、 7 种 PAHs 和 1 种糖类. 5 种一次源的源谱为: 植物 碎屑采用 Rogge 等 ${ }^{[28]}$ 的结果, 木材燃烧采用 Fine 等 ${ }^{[29]}$ 的结果, 汽油车和柴油车采用 Schauer 等 ${ }^{[30,31]}$ 的研究结 果, 燃煤采用 Zheng 等 ${ }^{[26]}$ 的研究结果. 模型运行结果要 求拟合相关系数 $R^{2}>0.85$, Chi-square $\chi^{2}<4$, 解析结果 不满足条件的不参与计算. 表 2 给出了本研究中 $\mathrm{CMB}$ 模型解析得到的各种一次源对 $\mathrm{OC}$ 的贡献. CMB 方法未 解析出的部分在北大点和榆仼点分别占 $\mathrm{OC}$ 的 $60 \%$ 和 $59 \%$, 认为这部分主要为二次生成的贡献.

表 1 二次有机示踪物浓度 $\left(\mathrm{ng} / \mathrm{m}^{3}\right)$

Table 1 Concentrations of SOA tracers as KPA $\left(\mathrm{ng} / \mathrm{m}^{3}\right)$

\begin{tabular}{|c|c|c|c|c|c|}
\hline \multirow{2}{*}{ Compound } & \multicolumn{2}{|c|}{ PKU } & \multicolumn{2}{|c|}{ Yufa } & \multirow{2}{*}{ Precursor } \\
\hline & Average & Stdev & Average & Stdev & \\
\hline 2-Methylglyceric acid & 21.0 & 19.5 & 29.9 & 19.9 & Isoprene \\
\hline 2-Methylthreitol & 42.2 & 28.2 & 63.6 & 46.8 & Isoprene \\
\hline 2-Methylerythritol & 77.2 & 60.2 & 121.5 & 101.4 & Isoprene \\
\hline Sisoprene & 140.4 & 100.9 & 215.0 & 160.3 & \\
\hline 3-Isopropylpentanedioic acid & 8.7 & 7.1 & 9.6 & 7.2 & $\alpha$-pinene \\
\hline 3-Acetylpentanedioic acid & 6.8 & 7.1 & 5.3 & 2.8 & $\alpha$-pinene \\
\hline 3-methyl-1,2,3-butanetricarboxylic acid & 4.6 & 4.0 & 4.3 & 6.9 & $\alpha$-pinene \\
\hline 3-Acetylhexanedioic acid & 8.3 & 5.8 & 7.7 & 5.5 & $\alpha$-pinene \\
\hline 3-Hydroxyglutaric acid & 51.7 & 31.3 & 57.5 & 27.4 & $\alpha$-pinene \\
\hline 2-Hydroxy-4,4-dimethylglutaric acid & 9.9 & 9.1 & 8.3 & 6.7 & $\alpha$-pinene \\
\hline 3-(2-Hydroxy-ethyl)-2,2-dimethyl cyclobutane-carboxylic acid & 8.5 & 10.1 & 6.1 & 5.5 & $\alpha$-pinene \\
\hline Pinic acid & 3.2 & 6.4 & 3.4 & 5.7 & $\alpha$-pinene \\
\hline Pinonic acid & 11.9 & 6.3 & 21.9 & 9.2 & $\alpha$-pinene \\
\hline$\Sigma \alpha$-pinene & 113.5 & 63.3 & 124.1 & 47.8 & \\
\hline 2,3-Dihydroxy-4-oxopentanoic acid & 13.3 & 7.7 & 11.7 & 6.9 & Toluene \\
\hline$\beta$-Caryophyllinic acid & 5.1 & 4.5 & 3.6 & 2.3 & $\beta$-Caryophyllene \\
\hline
\end{tabular}


表 2 一次源对 PM2.5 中 $\mathrm{OC}$ 的贡献(平均值土标准偏差, \%)

Table 2 Source contributions to measured PM2.5 OC (average \pm standard deviation, \%)

\begin{tabular}{|c|c|c|c|c|c|c|}
\hline Site & Gasoline vehicle & Diesel vehicle & Vegetative detritu & Biomass burning & Coal burning & Other OC \\
\hline PKU & $10.3 \pm 8.7$ & $16.2 \pm 5.9$ & $1.4 \pm 0.8$ & $7.5 \pm 5.5$ & $5.8 \pm 5.5$ & $60.7 \pm 13.2$ \\
\hline Yufa & $7.9 \pm 6.2$ & $14.5 \pm 4.1$ & $1.9 \pm 1.5$ & $8.9 \pm 4.9$ & $7.8 \pm 4.6$ & $58.8 \pm 11.0$ \\
\hline
\end{tabular}

2.1.3 非生物质燃烧水溶性有机碳方法(Non-Biomass burning WSOC)

大气颗粒物中的水溶性有机物(WSOC)主要有两个 来源: 生物质燃烧和二次转化 ${ }^{[32]}$. 因此用大气颗粒物中 总的 WSOC 去掉生物质燃烧排放的 WSOC 就可以粗略 地估算出大气中 SOC 的含量. 虽然这种方法的不确定 性较大, 但是由于方法简单, 因此应用中仍然有一定的 实用价值. Eq. 3 为非生物质燃烧 WSOC 法的计算公式.

$$
\mathrm{SOC}=\mathrm{WSOC}-\mathrm{OC}_{\mathrm{BB}} R_{\mathrm{w} / \mathrm{o}}
$$

式中 WSOC 为颗粒物中总的水溶性有机碳, $\mathrm{OC}_{\mathrm{BB}}$ 为生 物质燃烧排放产生的 $\mathrm{OC}$, 可以由 $\mathrm{CMB}$ 模型得到. 该方 法中最重要的参数为 $R_{\mathrm{w} / \mathrm{o}}$, 即生物质然烧颗粒物中水溶 性组分占总 $\mathrm{OC}$ 的比例.

已经有一些研究者对生物质然烧颗粒物中 WSOC 和 OC 的比值进行了测量. 例如 Peltier 等 ${ }^{[33]}$ 利用利用大 气颗粒物在线水萃取采样仪(PILS)与 WSOC 分析仪联 用或 OC 分析仪联用在生物质燃烧期间对颗粒物中水溶 性有机碳和总碳进行测量, 由于在观测期间生物质燃烧 对大气颗粒物的贡献非常大，可以认为在该期间测定的 颗粒物主要由生物质燃烧产生, 其他的来源可以忽略不 计. 由此得到生物质燃烧产生的颗粒物中 WSOC/OC 的 比值为 $0.56 \pm 0.05$. 但是这个比值由于不同地区使用不 同的生物质不同而有所差异, 因此需要针对我国生物质 燃烧的参数.

本研究对我国典型的生物质进行模拟燃烧, 以获得 非生物质然烧 WSOC 法估算的参数, 模拟实验在模拟 燃烧室中进行. 模拟燃烧室的具体设计和参数在 $\mathrm{He}$ 等 ${ }^{[34]}$ 的文章中有详细描述. 模拟燃烧的生物质包括我 国南北方典型的秸秆、灌木和乔木(包括软木和硬木). 表 3 给出了本研究不同种类生物质燃烧颗粒物中 $\mathrm{WSOC} / \mathrm{OC}$ 的比值, 从表中可以发现, 我国不同的生物 质燃烧排放 WSOC 的排放因子变化较大, 甘庶和水稻 燃烧排放颗粒物中 WSOC 的排放因子分别为 $(0.37 \pm$ $0.09)$ 和(4.39 \pm 3.29$) \mathrm{g} / \mathrm{kg}$; 而木材燃烧中 WSOC 的排放 因子变化范围为从 0.89 到 $2.69 \mathrm{~g} / \mathrm{kg}$. 尽管不同生物质排 放颗粒物中 WSOC 的排放因子变化非常大, 但是 WSOC 占 OC 的比值却变化不大. 表 3 给出了本研究模 拟生物质燃烧产生颗粒物中 WSOC与 OC 的比值. 从表 中可以看出, 秸秆燃烧中 WSOC/OC 的比值略小, 分别 为甘庶 $0.44 \pm 0.04$, 水稻 $0.45 \pm 0.04$, 而木材燃烧中的这 个比值在 $0.48 \sim 0.49$ 之间, 所有生物质的平均值为 0.48 \pm 0.04 , 因此本研究采用的非生物质燃烧 WSOC 法的表 达公式为:

$$
\mathrm{SOC}=\mathrm{WSOC}-0.48 \times \mathrm{OC}_{\mathrm{BB}}
$$

利用 Eq. 4 以及 CMB 模型获得的生物质燃烧贡献，对北 大点和榆货点观测期间的 SOC 进行估算. 观测期间两 个站点 SOC 都可以占到 OC 很高的比例. 榆华点 SOC 的浓度范围为 $1.74 \sim 17.11 \mu \mathrm{g} / \mathrm{m}^{3}$, 平均浓度为 $(6.32 \pm$ 2.90) $\mu \mathrm{g} / \mathrm{m}^{3}$; 北大点 SOC 的浓度要低于榆袋点, 浓度范 围为 $1.97 \sim 10.75 \mu \mathrm{g} / \mathrm{m}^{3}$, 平均浓度为 $(5.24 \pm 1.71) \mu \mathrm{g} / \mathrm{m}^{3}$. 观测期间榆佳点 SOC 所占 OC 比例平均为(52.6土 $17.4) \%$, 对应的北大点为 $(46.2 \pm 12.5) \%$.

\subsubsection{EC 示踪 OC/EC 比值法}

元素碳(EC)主要来自化石燃料和生物质的不完全 燃烧，因此可以作为一次排放碳质气溶胶的示踪物 ${ }^{[35]}$. $\mathrm{EC}$ 示踪 OC/EC 比值法是应用非常广泛的二次有机气溶 胶的估算方法. OC/EC 比值法由于只需要利用外场观测 的数据, 具有方法简单和时间分辨率高等优点.

$\mathrm{EC}$ 示踪法估算 $\mathrm{SOC}$ 最重要的就是确定一次源排放 $\mathrm{OC} / \mathrm{EC}$ 的比值, Lin 等 ${ }^{[36]}$ 对北京市大气颗粒物中 $\mathrm{EC} / \mathrm{OC}$ 的研究表明, 由于 $\mathrm{OC}$ 和 $\mathrm{EC}$ 的排放源之一交通源存在 昼夜的差异，因此在白天和夜间一次源排放 $\mathrm{OC} / \mathrm{EC}$ 的 比值是变化的, 而且由于示踪物产率法和 $\mathrm{CMB}$ 方法的 采样时间分辨率为昼夜采样, 为了更好地将 $\mathrm{OC} / \mathrm{EC}$ 比 值法和这两种方法进行比较, 因此本研究在估算 SOC 时将白天和夜间分开计算一次源排放 OC/EC 比值. 根 据 $\Delta \mathrm{EC} / \Delta \mathrm{CO}$ 比值的日变化规律, 将白天定义为每天的 9:00 22:00, 夜间从 23:00 至次日 8:00.

分别对白天和夜间的大气颗粒物中的 $\mathrm{OC} / \mathrm{EC}$ 比值 进行频率分布统计, 选取 OC/EC 比值较小的 OC, EC 观 测数据的子集，并对这个子集的数据作线性回归来得到 一次源 $\mathrm{OC} / \mathrm{EC}$ 的比值. 对白天数据, 我们选用 $\mathrm{OC} / \mathrm{EC}$ 比值的 $10 \%$ 分位数(即 $\mathrm{OC} / \mathrm{EC}$ 比值当中较小的那 $10 \%$ 的 $\mathrm{OCEC}$ 数据) 作为 $(\mathrm{OC} / \mathrm{EC})_{\mathrm{pri}}$ 的上限, 回归得到的 $(\mathrm{OC} / \mathrm{EC})_{\text {pri }}$ 比值为 1.46 ; 对于夜间数据, 考虑到夜间由 于缺少太阳辐射, 光化学反应相对于白天要微弱许多, 因此选用 $\mathrm{OC} / \mathrm{EC}$ 比值的 $20 \%$ 分位数, 这相当于给夜间 赋予了较高于白天的一次排放的权重, 计算得到 $(\mathrm{OC} / \mathrm{EC})_{\mathrm{pri}}$ 比值为 1.0. 利用这一比值, $\mathrm{OC} / \mathrm{EC}$ 比值法获 得的北大点 SOC 占总 $\mathrm{OC}$ 的 $(58.4 \pm 17.1) \%$.

图 2 汇总了本研究中 4 种估算方法得到的北大站点 SOC 占总 OC 的百分比, 解析的结果存在较大的差别, 按照百分比从小到大的顺序依次为示踪物 - 产率法 $<$ 非 
表 3 生物质燃烧颗粒物中 WSOC/OC 比值

Table 3 Mass ratios of WSOC/OC in biomass burning particles

\begin{tabular}{cccccccccc}
\hline & Sugarcane leave & Rice leave & Southern cedar & Banyan & Pine & Willow & Southern frutex & Northern frutex & average \\
\hline Average & 0.44 & 0.45 & 0.48 & 0.48 & 0.48 & 0.49 & 0.49 & 0.49 & 0.48 \\
Std & 0.04 & 0.04 & 0.04 & 0.05 & 0.05 & 0.03 & 0.04 & 0.05 & 0.04 \\
\hline
\end{tabular}

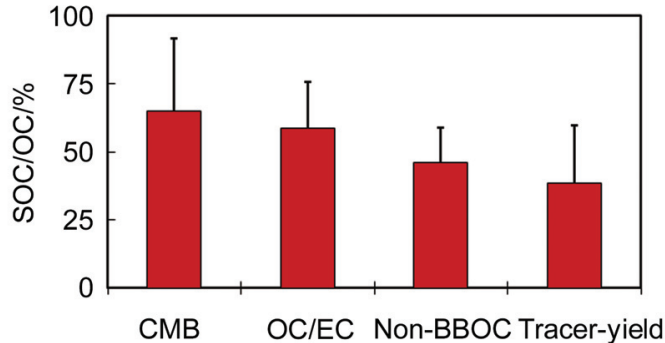

图 2 各种 SOC 估算方法得到的 SOC 占 $\mathrm{OC}$ 的百分比

Figure 2 Mass fraction of estimated SOC to total OC

生物质然烧 WSOC 法 $<\mathrm{OC} / \mathrm{EC}$ 比值法 $<$ 非一次源 $\mathrm{OC}$ 法(CMB 方法), 这样的结果也表明了不同方法得到的 SOC 所代表 SOC 的程度. 但是无论何种方法, 颗粒有机 物中二次来源都占有很高的比例, SOC 占总 $\mathrm{OC}$ 的比例 大约在 50\%甚至更高, 说明二次转化已经成为北京夏季 颗粒有机物的重要来源.

\section{2 示踪物法解析颗粒有机物一次源与二次源的闭合}

CMB 方法和示踪物产率法利用示踪物对颗粒有机物进 行一次源和二次源解析, 示踪物法的优点在于利用示踪 物对源的指纹指示特征可以解析出特定的一次和二次 源的贡献. 本研究综合两种方法对总 $\mathrm{OC}$ 进行闭合, 目 的在于弄清基于目前的技术对颗粒有机物来源的认识 程度. 图 3 给出了观测期间两个站点各种一次源和二次 源对总 $\mathrm{OC}$ 的平均贡献. 从图中可以看出, $\mathrm{CMB}$ 方法和 示踪物产率法解析出的一次源和二次源在北大点可以 解释 $(72.2 \pm 9.6) \%$ 的 $\mathrm{OC}$; 在榆栠点, 两种方法可以解释 $(80.2 \pm 10.5) \%$ 的 OC. 在北大点未解析出的 OC 要略高, 这主要是由于北大点颗粒物的来源相对会比较复杂, 可 能存在更多未解析出的一次源; 而榆仼点未解析的部分 很有可能主要来自于其他二次源的贡献. 根据闭合的结

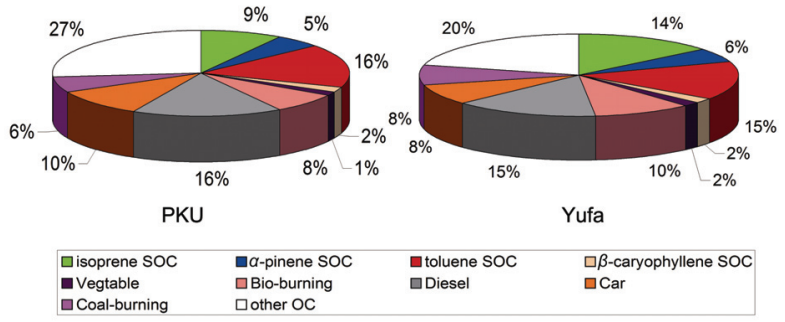

图 $3 \mathrm{CMB}$ 法和示踪物产率法解析颗粒有机物一次/二次来源对总 $\mathrm{OC}$ 的闭合

Figure 3 Closure of primary and secondary organic carbon from CMB and tracer yield method to total particulate OC
果可以看出，就目前的源解析技术而言，在两个站点仍 然有 $20 \% \sim 27 \%$ 的 OC 来源并不清楚.

\section{$2.3 \mathrm{SOC}$ 估算方法的比较}

2.3.1 示踪物一产率法、非一次源 OC 法和 OC/EC 比值 法估算 SOC

示踪物-产率法、非一次源 OC 法和 $\mathrm{EC}$ 示踪 $\mathrm{OC} / \mathrm{EC}$ 比值法都能够对大气中的 SOC 进行估算, 综合比较几 种方法的结果一方面有助于验证结果的可靠性以及方 法中参数设定的合理性, 另外可以对这几种方法的不确 定性有所认识.

图 4 给出了北大点三种方法估算 SOC 的时间序列. 总体来看三种方法估算的 SOC 在整个观测期间的变化 趋势比较一致. 对这三种方法估算得到的 SOC 进行相 关分析(图 5). 从图 5a 中可以看出示踪物-产率法和 $\mathrm{CMB}$ 方法估算得到的 SOC 相关性很好, 相关系数 $r=$ $0.86(n=75)$, 大于在自由度为 $73(n-2)$ 、显著性水平为 $5 \%$ 的条件下 $p$ 值 0.23 , 说明两组数据显著相关. 另外, 回归曲线的斜率为小于 1 , 表明 CMB 方法估算的 SOC 比示踪物-产率法系统偏高. 从图 $5 \mathrm{~b}, 5 \mathrm{c}$ 可以看出, $\mathrm{CMB}$ 方法和 $\mathrm{EC}$ 示踪 $\mathrm{OC} / \mathrm{EC}$ 比值法以及示踪物-产率法和 $\mathrm{EC}$ 示踪 $\mathrm{OC} / \mathrm{EC}$ 比值法的相关系数分别为 $r=0.58$ 和 0.55 $(n=65)$, 也大于在自由度为 $63(n-2)$ 、显著性水平为 $5 \%$ 条件下的 $p$ 值 0.27 , 因此两组数据显著相关.

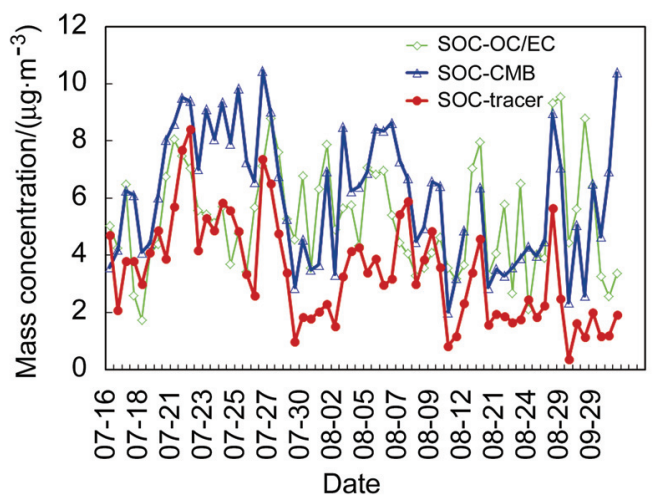

图 $4 \mathrm{EC}$ 示踪 $\mathrm{OC} / \mathrm{EC}$ 比值法、非一次源 $\mathrm{OC}$ 法和示踪物产率法估算 SOC 比较

Figure 4 Comparison of SOC from EC tracer EC/OC ratios method, CMB modeling and tracer yield method

研究结果表明，这几种方法都可以适用于北京大气 中二次有机气溶胶的估算，但在使用时需要注意不同方 法之间存在的系统误差. 

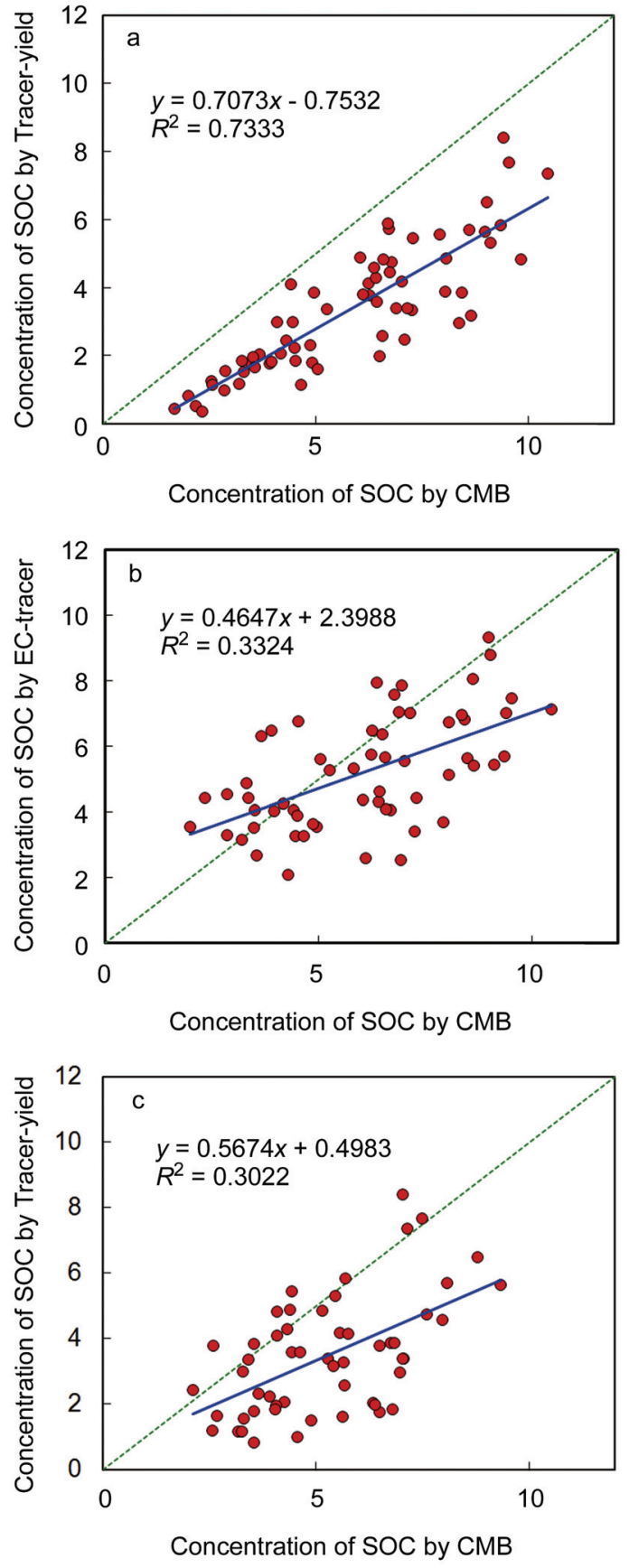

图 5 示踪物产率法、 $\mathrm{CMB}$ 法和 $\mathrm{EC}$ 示踪 $\mathrm{OC} / \mathrm{EC}$ 比值法估算 $\mathrm{SOC}$ 的 相关分析

Figure 5 Correlations of SOCs between tracer yield method, CMB and EC tracer OC/EC ratio method

(a) tracer yield/CMB, (b) $\mathrm{CMB} / \mathrm{OCEC}$ ratio, (c) tracer yield/OCEC ratio

\subsection{2 非生物质燃烧 WSOC 法和 OC/EC 比值法估算} $\mathrm{SOC}$

本研究对非生物质燃烧 WSOC 法和 EC-示踪 OC/EC 比值法估算的 SOC 进行了比较. 相关分析的结 果发现, 两种方法估算的 SOC 相关性比较差, 相关系数 $\mathrm{r}$ 仅为 0.1. 在国外的研究中也发现, 这两种方法估算的 结果差别很大 ${ }^{[37]}$. 造成这一结果的主要原因是非生
物质燃烧 WSOC 方法估算得到的是水溶性的 SOC, 而水溶性的 SOC 占总 SOC 的比值会随着时间和地点 的不同而变化. Kondo 等 ${ }^{[38]}$ 通过直接比较 PILS-WSOC 和 AMS 解析出的 SOC 估算得到在东京 大概 $6 \% \sim 26 \%$ 的 SOC 为非水溶性的, 这个百分比在 不同地区和不同季节都会有所变化，甚至在同一天 的不同时段都存在很大的变化. 在本研究中, 通过比 较非生物质燃烧 WSOC 法和 OC/EC 比值法的结果, 得到在北京夏季水溶性的 SOC 可以占到 SOC 的(86 $\pm 29) \%$ ，也就是说有大约 $14 \%$ 的非水溶性的 SOC 没 有被考虑. 更重要的是这个比值在不同时段的变化 也较大，因此非生物质燃烧 WSOC 法仅仅可以对北 京大气颗粒物中的水溶性 SOC 进行估算.

\subsection{SOC 估算方法的不确定性分析}

本研究通过比较多种 SOC 的估算方法, 初步地对 各种方法的不确定性进行分析. 本研究仅仅针对估算方 法原理的本身进行不确定性的讨论，因此并不是精确的 定量分析, 目的只是为让研究者在使用这些方法时对结 果的不确定性有定性的认识.

本研究不确定性分析主要包括两个方面，一是方法 本身的一些参数设定对估算结果的影响; 二是估算得到 的 SOC 对真实 SOC 的代表程度. 单点测定的不确定性 按照 Eqs. 5, 6 计算得到.

当测定值低于最低检测限(MDL)时，采用公式:

$$
U n c=\frac{5}{6} \mathrm{MDl}
$$

当测定值大于最低检测限(MDL)时，采用公式：

$$
U n c=\sqrt{(\text { ErrorFraction } \times \text { concentration })^{2}+(\mathrm{MDL})^{2}}
$$

公式中 $U n c$ 为不确定性, MDL 为最低检测限.

在不确定性分析中将使用三个参数：第一个是估算 中一些参数的设定导致的不确定性(以下称为参数不确 定性 $U_{\mathrm{p}}$ ), 对这个不确定性的讨论有助于对方法的进一 步改进; 第二个是计算方法中由于方法的参数设置、仪 器测量以及估算过程的运算中造成的不确定性，称为估 算不确定性 $\left(U_{\mathrm{c}}\right)$, 用绝对误差 $\left(U_{\mathrm{ca}}\right)$ 和相对误差 $\left(U_{\mathrm{cr}}\right)$ 表 示, 这个误差也能够全面的反映估算本身在运算过程中 造成的不确定性; 第三个是由于估算原理造成的结果对 估算预期的低估或者高估程度( 以下称为估算偏差 $E$ ), 这个不确定性是用来评价估算原理所带来的不确定性.

(1)示踪物-产率法

示踪物产率法能够准确的估算某一特定 VOCs 前体 物生成 SOC 的含量. 从严格意义上讲，只有示踪物产率 法是真正解析特定来源(VOCs 前体物)对 SOA 贡献的方 法.

示踪物产率法最重要的参数(即 $f_{\mathrm{soa}}$ 和 $f_{\mathrm{soc}}$ ) 是来自于 烟雾箱实验的结果，实际大气中的情况是十分复杂的， 利用烟雾箱实验获得的参数是很难模拟实际大气中的 
情况的, 因此实际大气与烟雾箱中的差别是造成这种方 法不确定性的最大方面. 减少这种不确定性的方法是增 加更多的示踪物, 当示踪物种类非常少时, 每种示踪物 对于整个结果的敏感性就会增加, 因此增加估算结果的 不确定性. 例如甲苯 SOC 的示踪物只有一种, 因此对甲 苯 SOC 示踪物的测量有微小的变化, 估算结果的误差 也会被放大. 这种由于参数造成的不确定性可以用烟雾 箱实验中对该参数测定的随机误差即测定比值的标准 偏差表示, 因此参数不确定性分别为异戊二烯 $25 \% 、 \alpha-$ 蒎烯 $48 \%$ 、 $\beta$-丁香烯 $20 \%$ 、甲苯 $33 \%$.

根据 Kleindienst 等的边界分析(boundary analysis) 的结果表明，估算结果对 SOC 的贡献范围(置信区间)为 异戊二烯 $70 \% \sim 130 \% 、 \alpha$-蒎烯 50\% 220\%、 $\beta$-丁香烯 $70 \% \sim 120 \%$ 、甲苯 $60 \% \sim 160 \%{ }^{[23]}$. 综合测量和计算所 带来的误差, 示踪物产率法对异戊二烯、 $\alpha$-蒎烯、 $\beta$-丁 香烯和甲苯生成 SOC 估算的绝对误差分别为(184.6土

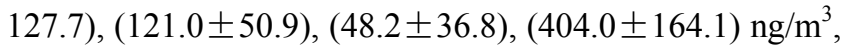
相对误差为 $(21.8 \pm 4.0) \%,(27.6 \pm 7.7) \%,(27.7 \pm 13.7) \%$, $(28.5 \pm 8.3) \%$. 对解析出的总 SOC 绝对计算误差为 $(1.12$ $\pm 0.57) \mu \mathrm{g} / \mathrm{m}^{3}$ ，相对误差为 $(24.8 \pm 5.7) \%$.

从代表程度上来看, 这种方法估算出的 SOC 是真 实 $\mathrm{SOC}$ 的一部分, 因此是真实 $\mathrm{SOC}$ 的一个子集. 假定 $\mathrm{EC}$ 示踪 $\mathrm{OC} / \mathrm{EC}$ 比值法的结果作为预期, 与之相比较, 示踪物-产率法对 SOC 的估算偏差 $E$ 大约为低估了 $(44.0$ $\pm 21.1) \%$.

\section{(2)非生物质燃烧 WSOC 法}

这种方法从 WSOC 的来源入手, 误差主要来自两 个假定: 一是认为 SOC 大部分为水溶性的; 二是仅考虑 WSOC 的两个来源二次转化和生物质燃烧.

从本研究的结果看, 我国生物质燃烧排放颗粒物中, WSOC 与 OC 的比值是比较稳定的, 由这个比值带来的 不确定性为 $8 \%$. 该方法的不确定性主要来自非水溶性 的 SOC, 但是由于非水溶性 SOC 占 OC 的比例具有非常 大的变化, 无法用简单的系数对其进行校正. 另外其他 源排放的 WSOC 例如工业和机动车源等也会对结果造 成误差. 综合测量和参数的误差, 非生物质燃烧 WSOC 法的估算不确定性为: 绝对误差 $(1.02 \pm 0.35) \mu \mathrm{g} / \mathrm{m}^{3}$, 相 对误差 $(19.3 \pm 0.8) \%$.

从代表性上来看, 该方法得到的是水溶性的 $\mathrm{SOC}$, 是真实 $\mathrm{SOC}$ 的一个子集. 前面也已经提到, 与 $\mathrm{OC} / \mathrm{EC}$ 比值法相比较, 非生物质燃烧 WSOC 法对 SOC 的估算 偏差大约为低估了 $(14.0 \pm 20.7) \%$, 估算偏差较大的标准 偏差(20.7\%)也表明水溶性 SOC 在 SOC 中所占比例变化 很大.

\section{(3)非一次源 OC 法(CMB 方法)}

这种方法最基本的思想是受体模型 CMB 方法没有 解析出的部分即为二次源贡献. 因此方法的原理本身就 意味着该方法只是粗略的估算.
$\mathrm{CMB}$ 方法的参数不确定性主要来自源谱, 通过敏 感性分析(sensitivity analysis)获得. 敏感性分析是通过 输入 4 种不同的生物质燃烧源谱, 分析源解析结果的稳 定性，这 4 种源谱包括一种开放式燃烧 [39]、两种火炉燃 烧 ${ }^{[25,29]}$ 和一种壁炉燃烧 ${ }^{[40]}$. 研究结果发现, 4 种源谱获 得的生物质燃烧贡献的变化因子在 $0.75 \sim 1.30$ 之间, 差 别最大的是使用开放式燃烧源谱, 其他源谱造成的结果 波动在 1.09 以内. 考虑到北京地区严格限制开放式燃烧, 因此认为源谱选择带来的误差小于 $10 \%$. 综合运算所带 来的误差, $\mathrm{CMB}$ 方法对二次源估算的计算不确定性绝 对误差为 $(0.77 \pm 0.47) \mu \mathrm{g} / \mathrm{m}^{3}$ ，相对误差为 $(12.6 \pm 5.3) \%$.

从代表程度上来看，该方法解析的结果包括了全部 的 SOC 还有部分没有解析出的一次源 OC (POC). 例如 已有的研究表明，餐饮排放也是北京大气颗粒有机物的 重要来源 ${ }^{[25,41]}$. 但是在本研究中, 作为餐饮排放的示踪 物胆固醇类物质浓度很低，而且将餐饮源进入计算会使 得模型的运行结果不稳定, 使北大点 74 个样品中的 32 个和榆仼 99 个样品中的 21 个不能达到模型运行要求, 因此本研究未考虑餐饮排放. 但是, 如果餐饮排放的确 对颗粒有机物有贡献，其贡献将会被解析到其他 OC 当 中, 因此本研究中非一次源 OC 法获得的 SOC 是一个高 估的结果. 将 $\mathrm{OC} / \mathrm{EC}$ 比值法的结果作为真实 SOC 的预 期, 非一次源 OC 法估算的不确定性为高估了约(33.9土 $41.1) \%$.

(4)EC 示踪 OC/EC 比值法

这种方法利用 $\mathrm{EC}$ 作为一次源排放的示踪物, 用总 的 OC 减去一次源的 OC 即得到 SOC, 从代表的程度上, 估算结果预期得到真实大气中的 SOC. 在估算中, 该方 法最重要的就是要确定一次源排放 $\mathrm{OC} / \mathrm{EC}$ 的比值, 同 时也是该方法中最大的不确定性.

为获得 $\mathrm{OC} / \mathrm{EC}$ 比值法的参数不确定性, 本研究采 用 bootstrap 的方法, 对全部的白天或者晚上的 $\mathrm{OC}$ 和 $\mathrm{EC}$ 数据随机取样(可重复取样), 然后用随机取得的数据按 照前面提到的方法计算一次源 $\mathrm{OC} / \mathrm{EC}$ 比值，重复计算 100 次, 得到的 100 个一次源 $\mathrm{OC} / \mathrm{EC}$ 比值的标准偏差即 为参数不确定性. 利用这种方法得到参数不确定性为 $(28.5 \pm 21.6) \%$. 得到这个参数不确定性后, 就可以利用 误差传递公式，对整个计算的不确定性进行估算，最后 得到该方法计算的不确定性为绝对误差 $(0.65 \pm 0.44)$ $\mu \mathrm{g} / \mathrm{m}^{3}$, 相对误差为 $(11.3 \pm 3.8) \%$.

对于 $\mathrm{OC} / \mathrm{EC}$ 法的估算偏差, 本研究中白天采用 $10 \%$ 最小值、夜间采用 $20 \%$ 最小值作为一次源 $\mathrm{OC} / \mathrm{EC}$ 比值可能高估白天的 SOC 或低估夜间的 SOC. 与 CMB 方法比较, 从图 $5 \mathrm{~b}$ 中可以看出, 少数情况下 $\mathrm{CMB}$ 方法 的结果偏低, 而且这种情况大部分出现在白天的数据中 造成这种情况的原因可能是由于本研究中 $\mathrm{OC} / \mathrm{EC}$ 比值 法白天选择较低的一次源 $\mathrm{OC} / \mathrm{EC}$ 比值, 这样可能造成 $\mathrm{SOC}$ 的高估. 这种高估在单点可能大于 $54 \%$. 与示踪物 
产率法相比, 大多数情况下, $\mathrm{OC} / \mathrm{EC}$ 比值法估算的结果 要高于示踪物-产率法, 如图 $5 \mathrm{c}$ 所示. 但是, 也有少数 情况 OC/EC 比值法偏低, 这种情况大部分出现在夜间 数据, 这有可能是由于夜间选取较高一次源 $\mathrm{OC} / \mathrm{EC}$ 比 值造成的. 这种低估最大可能大于 $64 \%$. 由于真实的一 次源 $\mathrm{OC} / \mathrm{EC}$ 比值不可知, 而且变化很大, 因此降低这种 不确定性的方法是增加数据量, 从统计学上找到更加合 理的方法获得一次源 $\mathrm{OC} / \mathrm{EC}$ 比值.

表 4 汇总了示踪物产率法、非生物质燃烧 WSOC 法、 $\mathrm{EC}$ 示踪 OC/EC 比值法和非一次源 $\mathrm{OC}$ 法的不确定性分 析的结果. 需要注意的是, 估算不确定性表征的是估算 结果对该方法本身估算预期的不确定性, 并不是对总 $\mathrm{SOC}$ 的预期. 例如 $\mathrm{OC} / \mathrm{EC}$ 比值法的估算误差很大, 为 $(43.0 \pm 4.9) \%$, 但是其表征的是对总 SOC 估算的误差; 相 反地, 非生物质燃烧 WSOC 法的估算不确定性虽然很小, 为(19.3 \pm 0.8$) \%$, 但是其表征的是对水溶性 SOC 的估算 误差为 $(19.3 \pm 0.8) \%$. 因此研究者在选取方法时要综合考 虑这些估算不确定性和估算偏差以及研究的目的.

\section{3 结论}

利用多种方法对 CAREBEIJING 2008 年夏季大型 观测期间 PM2.5 中的二次有机气溶胶进行估算. 估算结 果发现, 二次有机气溶胶是颗粒有机物中的重要组成部 分, SOC 占 OC 的比例可以达到 $50 \%$ 甚至更高. 但是不 同方法的估算结果之间存在很大的差别. 利用有机示踪 物法, 包括 CMB 模型解析颗粒有机物一次来源和示踪 物产率法解析颗粒物二次来源, 对颗粒有机物来做闭合, 研究发现以目前的示踪物技术, 北京大气颗粒有机物中 仍然有 $20 \% \sim 27 \%$ 的来源不清楚. 本研究还综合比较了 目前国际上应用较多的多种 SOC 估算方法, 其中包括 示踪物产率法、非一次源 $\mathrm{OC}$ 法 $(\mathrm{CMB})$ 、非生物质燃烧 水溶性有机碳(WSOC)方法和 EC 示踪 OC/EC 比值法, 研究结果表明示踪物产率法、CMB 方法和 EC 示踪 $\mathrm{OC} / \mathrm{EC}$ 比值法估算的 SOC 趋势一致, 因此都可以作为 我国 SOC 估算的方法, 但是需要注意不同方法之间存 在的系统误差. 而非生物质燃烧 WSOC 法仅能用来对 水溶性 SOC 进行估算. 本研究还对这几种方法的不确 定性做了初步的分析. 结果表明, SOC 估算的主要不确 定性来自于参数的设定和原理本身预期得到的 SOC 对
总 SOC 的表征程度. 研究者在选取估算方法时应综合 考虑方法的估算不确定性和估算偏差以及研究的目的.

\section{4 实验部分}

\section{1 采样点和采样时间}

本研究作为 CAREBEIJING 2008 (Campaigns of Air Quality Research in Beijing and Surrounding Region)的一 部分, 于 2008 年 7 月 16 日 $~ 8$ 月 31 日在城市点(北京 大学点)和乡村点(榆垈点)采集大气细颗粒物 PM2.5 样 品 ${ }^{[6,20]}$. 北大点 $\left(39^{\circ} 59^{\prime} 21^{\prime \prime} \mathrm{N}, 116^{\circ} 18^{\prime} 25^{\prime \prime} \mathrm{E}\right)$ 位于北京大学 校园内理科 1 号楼顶, 北京大学地处中关村地区，位于 北京的西北部. 观测平台位于校园东部教学楼顶, 距地 面约 $20 \mathrm{~m}$ 左右, 周围没有明显的污染排放源. 榆佳点 $\left(39^{\circ} 30^{\prime} 49^{\prime \prime} \mathrm{N}, 116^{\circ} 18^{\prime} 15^{\prime \prime} \mathrm{E}\right)$ 在北大点正南方向约 $53 \mathrm{~km}$, 位于北京市大兴区的最南端, 采样点位于榆袋镇黄浦大 学楼顶, 距离地面约 $20 \mathrm{~m}$. 周围主要为植被和少量居民 区，无明显污染排放源.

\section{2 采样方法与分析}

\subsection{1 离线膜采样}

本研究使用的大气颗粒物采样仪器是天虹公司的 四通道采样器(TH-16A, 武汉天虹), 四个通道的切割粒 径均为 PM2.5, 每个通道的采样流量均是 $16.7 \mathrm{~L} / \mathrm{min}$. 第一通道使用 Teflon 膜, 用作质量浓度、水溶性离子组 分和水溶性有机碳的分析 ${ }^{[20,42,43]}$; 第二、三、四通道使 用石英膜，从第二通道的石英膜上切割 $1 \mathrm{~cm} \times 1.45 \mathrm{~cm}$ 的面积用作 ECOC 的分析, 第二通道剩余膜部分和第 三、四通道的石英膜用作颗粒有机物的分析.

\subsection{2 颗粒物化学组成分析}

颗粒有机物的分析主要包括样品前处理和气相色 谱质谱联用仪 (GC/MS) 分析两部分, 详细的分析过程在 文献中有详细的描述 ${ }^{[14,27]}$. 最终, 本研究共定量了 154 种颗粒有机物种, 其中包括 14 种二次有机示踪物, 各物 种回收率在 $70 \% \sim 115 \%$ 之间，仪器精密度为 $0 \sim 12 \%$, 标准物质相对标准偏差为 $0 \sim 8 \%$, 满足环境样品的分析 要求.

\section{2 .3 在线 $\mathrm{OC} / \mathrm{EC}$ 分析仪}

本研究在北大站点利用美国 Sunset 实验室的半连 续 OC\&EC 分析仪, 用作在线测量大气细颗粒物 PM2.5

表 4 各种 SOC 估算方法的不确定性分析 ${ }^{a}$

Table 4 Uncertainty analysis of SOC estimation methods

\begin{tabular}{lcccc}
\hline & \multirow{2}{*}{$U_{\mathrm{p}} / \%$} & \multicolumn{2}{c}{$U_{\mathrm{c}}$} & \multirow{2}{*}{$E$} \\
\cline { 3 - 4 } & & $U_{\mathrm{ca}}\left(\mu \mathrm{gg} \cdot \mathrm{m}^{-3}\right)$ & $U_{\mathrm{cr}} / \%$ & \\
\hline Tracer yield & $20 \sim 48$ & $0.76 \pm 0.33$ & $24.8 \pm 5.7$ & Underestimate $(44.0 \pm 21.1) \%$ \\
Non-Biomass burning WSOC & 8 & $1.12 \pm 0.57$ & $19.3 \pm 0.8$ & Underestimate $(14.0 \pm 20.7) \%$ \\
EC tracer OC/EC ratio & $28.5 \pm 21.6$ & $0.65 \pm 0.44$ & $43.0 \pm 4.9$ & Single value: overestimate max $54 \%$, underestimate max $64 \%$ \\
Non-primary OC (CMB) & $<10$ & $0.77 \pm 0.47$ & $12.6 \pm 5.3$ & Overestimate $(33.9 \pm 41.1) \%$ \\
\hline
\end{tabular}

${ }^{a} U_{\mathrm{p}}$ : uncertainty coursed by parameters, $U_{\mathrm{c}}$ : uncertainty coursed by parameter and calculation, $E$ : estimation error by estimation theory. 
中 $\mathrm{OC}$ 和 $\mathrm{EC}$ 浓度, 仪器时间分辨率为 $1 \mathrm{~h}$, 采样流速为 $8 \mathrm{~L} / \mathrm{min}$, 分析方法为 NIOSHTOT(热光透射法)方法, 升 温程序与文献中的相同 ${ }^{[36,44]}$, 分析时间为 $15 \sim 20 \mathrm{~min}$.

\section{References}

[1] Kanakidou, M.; Seinfeld, J. H.; Pandis, S. N.; Barnes, I.; Dentener, F. J.; Facchini, M. C.; Van Dingenen, R.; Ervens, B.; Nenes, A.; Nielsen, C. J.; Swietlicki, E.; Putaud, J. P.; Balkanski, Y.; Fuzzi, S.; Horth, J.; Moortgat, G. K.; Winterhalter, R.; Myhre, C. E. L.; Tsigaridis, K.; Vignati, E.; Stephanou, E. G.; Wilson, J. Atmos. Chem. Phys. 2005, 5, 1053.

[2] Tsigaridis, K.; Kanakidou, M. Atmos. Chem. Phys. 2003, 3, 1849.

[3] Ulbrich, I. M.; Canagaratna, M. R.; Zhang, Q.; Worsnop, D. R.; Jimenez, J. L. Atmos. Chem. Phys. 2009, 9, 2891.

[4] Goldstein, A. H.; Galbally, I. E. Environ. Sci. Technol. 2007, 41, 1514.

[5] Zhang, R. Y. Science 2010, 328, 1366.

[6] Zheng, J.; Hu, M.; Zhang, R.; Yue, D.; Wang, Z.; Guo, S.; Li, X.; Bohn, B.; Shao, M.; He, L.; Huang, X.; Wiedensohler, A.; Zhu, T. Atmos. Chem. Phys. 2011, 11, 7755.

[7] Khalizov, A. F.; Lin, Y.; Qiu, C.; Guo, S.; Collins, D.; Zhang, R. Environ. Sci. Technol. 2013, 47, 2254.

[8] Zhang, R. Y.; Khalizov, A. F.; Pagels, J.; Zhang, D.; Xue, H. X.; McMurry, P. H. Proc. Natl. Acad. Sci. U. S. A. 2008, 105, 10291.

[9] Seinfeld, J. H.; Pandis, S. N. Atmospheric Chemistry and Physics-from Air Pollution to Climate Change, John Wiley \& Sons, Inc., New York, 1998.

[10] Hallquist, M.; Wenger, J. C.; Baltensperger, U.; Rudich, Y.; Simpson, D.; Claeys, M.; Dommen, J.; Donahue, N. M.; George, C.; Goldstein, A. H.; Hamilton, J. F.; Herrmann, H.; Hoffmann, T.; Iinuma, Y.; Jang, M.; Jenkin, M. E.; Jimenez, J. L.; Kiendler-Scharr, A.; Maenhaut, W.; McFiggans, G.; Mentel, T. F.; Monod, A.; Prevot, A. S. H.; Seinfeld, J. H.; Surratt, J. D.; Szmigielski, R.; Wildt, J. Atmos. Chem. Phys. 2009, 9, 5155.

[11] Huang, X. F.; He, L. Y.; Hu, M.; Canagaratna, M. R.; Sun, Y.; Zhang, Q.; Zhu, T.; Xue, L.; Zeng, L. W.; Liu, X. G.; Zhang, Y. H.; Jayne, J. T.; Ng, N. L.; Worsnop, D. R. Atmos. Chem. Phys. 2010, $10,8933$.

[12] Sun, J. Y.; Zhang, Q.; Canagaratna, M. R.; Zhang, Y. M.; Ng, N. L.; Sun, Y. L.; Jayne, J. T.; Zhang, X. C.; Zhang, X. Y.; Worsnop, D. R. Atmos. Environ. 2010, 44, 131.

[13] Sun, Y. L.; Wang, Z. F.; Fu, P. Q.; Yang, T.; Jiang, Q.; Dong, H. B.; Li, J.; Jia, J. J. Atmos. Chem. Phys. 2013, 13, 4577.

[14] Guo, S.; Hu, M.; Guo, Q. F.; Zhang, X.; Zheng, M.; Zheng, J.; Chang, C. C.; Schauer, J. J.; Zhang, R. Y. Environ. Sci. Technol. 2012, 46, 9846.

[15] Jia, L.; Xu, Y. F. Acta Chim. Sinica 2010, 68, 2429. (贾龙, 徐永福, 化学学报, 2010, 68, 2429.)

[16] Lü, Z. F.; Hao, J. M.; Li, J. H.; Wu, S. Acta Chim. Sinica 2008, 66, 419. (吕子峰, 郝吉明, 李俊华, 武山, 化学学报, 2008, 66, 419.)

[17] Sun, T. L.; Wang, Y. D.; Zhang, C. X.; Sun, X. M.; Hu, J. T. Acta Chim Sinica 2011，69，1965. (孙廷利, 王玉东, 张晨曦, 孙孝敏, 胡敬田, 化学学报, 2011, 69, 1965.)

[18] Guo, S.; Hu, M.; Shang, D.; Guo, Q.; Hu, W. Acta Chim. Sinica 2014, 72, 145. (郭松, 胡敏, 尚冬杰, 郭庆丰, 胡伟伟, 化学学报, 2014, 72, 145.)
[19] Docherty, K. S.; Stone, E. A.; Ulbrich, I. M.; DeCarlo, P. F.; Snyder, D. C.; Schauer, J. J.; Peltier, R. E.; Weber, R. J.; Murphy, S. M.; Seinfeld, J. H.; Grover, B. D.; Eatough, D. J.; Jimenez, J. L. Environ. Sci. Technol. 2008, 42, 7655.

[20] Guo, S.; Hu, M.; Wang, Z. B.; Slanina, J.; Zhao, Y. L. Atmos. Chem. Phys. 2010, 10, 947.

[21] Hu, M.; Guo, S. IGAC Newsletter 2009, 42, 10.

[22] Offenberg, J. H.; Lewis, C. W.; Lewandowski, M.; Jaoui, M.; Kleindienst, T. E.; Edney, E. O. Environ. Sci. Technol. 2007, 41, 3972.

[23] Kleindienst, T. E.; Jaoui, M.; Lewandowski, M.; Offenberg, J. H.; Lewis, C. W.; Bhave, P. V.; Edney, E. O. Atmos. Environ. 2007, 41, 8288 .

[24] Hu, D.; Bian, Q.; Li, T. W. Y.; Lau, A. K. H.; Yu, J. Z. J. Geophys. Res., [Atmos.] 2008, 113 (D2206).

[25] Wang, Q.; Shao, M.; Zhang, Y.; Wei, Y.; Hu, M.; Guo, S. Atmos. Chem. Phys. 2009, 9, 8573.

[26] Zheng, M.; Salmon, L. G.; Schauer, J. J.; Zeng, L. M.; Kiang, C. S.; Zhang, Y. H.; Cass, G. R. Atmos. Environ. 2005, 39, 3967.

[27] Guo, S.; Hu, M.; Guo, Q.; Zhang, X.; Schauer, J. J.; Zhang, R. Atmos. Chem. Phys. 2013, 13, 8303.

[28] Rogge, W. F.; Hildemann, L. M.; Mazurek, M. A.; Cass, G. R.; Simoneit, B. R. T. Environ. Sci. Technol. 1993, 27, 2700.

[29] Fine, P. M.; Cass, G. R.; Simoneit, B. R. T. Environ. Eng. Sci. 2004, $21,387$.

[30] Schauer, J. J.; Kleeman, M. J.; Cass, G. R.; Simoneit, B. R. T. Environ. Sci. Technol. 1999, 33, 1578.

[31] Schauer, J. J.; Kleeman, M. J.; Cass, G. R.; Simoneit, B. R. T. Environ. Sci. Technol. 2002, 36, 1169.

[32] Weber, R. J.; Sullivan, A. P.; Peltier, R. E.; Russell, A.; Yan, B.; Zheng, M.; de Gouw, J.; Warneke, C.; Brock, C.; Holloway, J. S.; Atlas, E. L.; Edgerton, E. J. Geophys. Res., [Atmos.] 2007, 112 (D1302).

[33] Peltier, R. E.; Weber, R. J.; Sullivan, A. P. Aerosol Sci. Technol. 2007, 41, 1117.

[34] He, L. Y.; Lin, Y.; Huang, X. F.; Guo, S.; Xue, L.; Su, Q.; Hu, M.; Luan, S. J.; Zhang, Y. H. Atmos. Chem. Phys. 2010, 10, 11535.

[35] Turpin, B. J.; Huntzicker, J. J. Atmos. Environ. 1995, 29, 3527.

[36] Lin, P.; Hu, M.; Deng, Z.; Slanina, J.; Han, S.; Kondo, Y.; Takegawa, N.; Miyazaki, Y.; Zhao, Y.; Sugimoto, N. J. Geophys. Res., [Atmos.] 2009, 114 (D00G11).

[37] Snyder, D. C.; Rutter, A. P.; Collins, R.; Worley, C.; Schauer, J. J. Aerosol Sci. Technol. 2009, 43, 1099.

[38] Kondo, Y.; Miyazaki, Y.; Takegawa, N.; Miyakawa, T.; Weber, R. J.; Jimenez, J. L.; Zhang, Q.; Worsnop, D. R. J. Geophys. Res., [Atmos.] 2007, 112 (D01203).

[39] Lee, S.; Baumann, K.; Schauer, J. J.; Sheesley, R. J.; Naeher, L. P.; Meinardi, S.; Blake, D. R.; Edgerton, E. S.; Russell, A. G.; Clements, M. Environ. Sci. Technol. 2005, 39, 9049.

[40] Schauer, J. J.; Kleeman, M. J.; Cass, G. R.; Simoneit, B. R. T. Environ. Sci. Technol. 2001, 35, 1716.

[41] Zhao, Y. L.; Hu, M.; Slanina, S.; Zhang, Y. H. Environ. Sci. Technol. 2007, 41, 99.

[42] Huang, X. F.; Hu, M.; He, L. Y.; Tang, X. Y. Atmos. Environ. 2005, $39,2819$.

[43] Hu, M.; Zhang, J.; Wu, Z. J. Sci. China, Ser. B 2005, 48, 265.

[44] Hu, W. W.; Hu, M.; Deng, Z. Q.; Xiao, R.; Kondo, Y.; Takegawa, N.; Zhao, Y. J.; Guo, S.; Zhang, Y. H. Atmos. Chem. Phys. 2012, 12, 1811 . 